\title{
Scham und Würde in der Pflege
}

Am Anfang steht die Wahrnehmung Pflege und Scham sind untrennbar verbunden.

Die Rahmenbedingungen von Pflege erschweren die würdevolle Pflege, nämlich die

Pflege, die Anerkennung, Schutz, Zugehörigkeit und Integrität aller zu wahren weiß. Das

bedeutet als Erstes, die Scham wahrzunehmen, sie bei sich selber und bei anderen gel-

ten zu lassen, damit sie ihre schützende Funktion als Hüterin der Würde entfalten kann.

Der Blick hat bei der Scham eine große Bedeutung. Wenn wir uns schämen, wenden wir den Blick ab. Ein Zeichen, das von Pflegekräften als Schutzsuche interpretiert und respektiert werden kann (Symbolbild).

- Ursula Immenschuh -

\section{Zum Schämen}

Zeitungsschlagzeilen weisen in Verbindung mit Berichten von Pflegekräften immer wieder darauf hin, dass die Pflege in Deutschland in einer Schieflage ist: Unterbesetzung, schlecht oder nicht qualifiziertes Personal, Überstunden, Versorgung von mehr als 60 pflegebedürftigen Heimbewohnerinnen und -bewohnern in der Nacht usw. Auch in einer Studie des Deutschen Instituts für Pflegewissenschaft (dip) ist nachzulesen, wie gravierend der Fachkräftemangel in der Pflege ist und

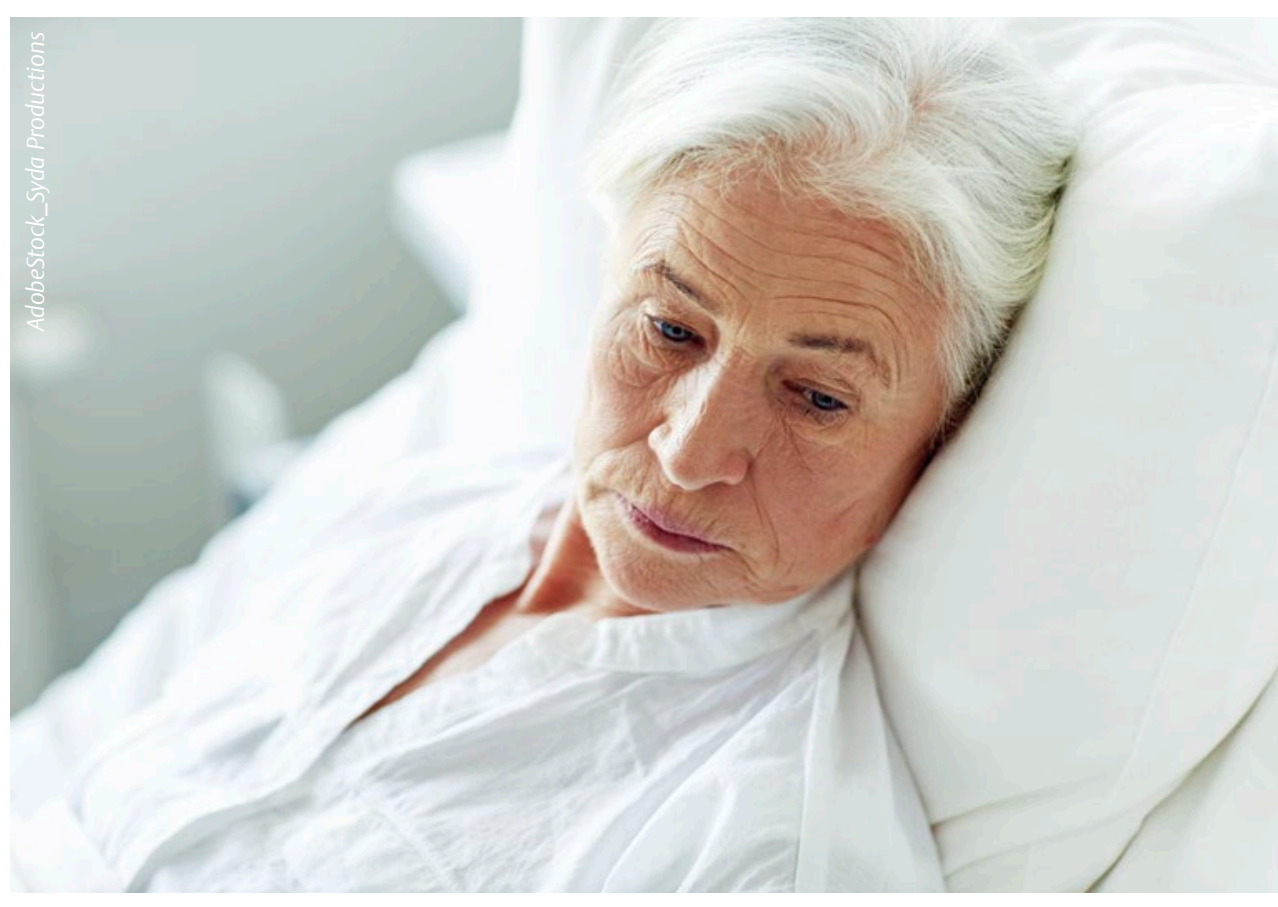

dass dieser Mangel direkte Auswirkungen auf die Pflegequalität hat. ${ }^{1}$

Wer sich die Diskussionen um Pflegebildung vor Augen führt, dem treibt es leicht die Schamesröte ins Gesicht. Während in den meisten Ländern

$$
\begin{aligned}
& \text { Würdevolle Pflege kann } \\
& \text { nicht innerhalb von } \\
& \text { Rahmenbedingungen } \\
& \text { geleistet werden, die } \\
& \text { unwürdig sind. }
\end{aligned}
$$

dass generalisierte Ausbildung zu teuer sei und dass akademisch ausgebildete Pflegekräfte womöglich zu viel Geld verlangen könnten. Dabei haben internationale Forschungen über 40 Jahre aus Magnetspitälern (und inzwischen auch Altenhilfeeinrichtungen sowie ambulanter Pflege) gezeigt, dass die Qualität der Pflege Menschen braucht, $\begin{array}{ll}\text { diskutiert man in Deutschland noch darü- } & \text { direkte Auswirkungen auf Mortalität und } \\ \text { ber, ob man Abitur braucht oder nicht, } & \text { Morbidität hat und dass bessere Qualität }\end{array}$ 
Die Scham hilft, Grenzen zu wahren - die der anderen und die eigenen Grenzen. Darum brauchen wir sie als Hüterin der Würde (Symbolbild).

nicht einmal teurer ist, wenn alle Folgekosten eingerechnet werden. ${ }^{2}$

Wer schämt sich also, wenn es um Pflege geht? Schämen sich die in der Politik Verantwortlichen? Die Betreiber von Heimen, die Gewinne einstreichen? Die Führungskräfte, die den Spagat zwischen Erfüllung der gesetzlichen Vorgaben und Unter- oder Fehlbesetzung täglich aushalten (müssen)? Sind es die Pflegekräfte, die Prioritäten setzen müssen, wem sie wie viel von ihrer Zeit ge-

ben und wem nicht? Oder schämen wir uns alle dafür, dass wir die Menschen, die der

$$
\begin{aligned}
& 99 \text { Die Scham ist die } \\
& \text { Hüterin der Würde. } \\
& \text { León Wurmser (1997) }
\end{aligned}
$$

Würde in Gefahr gerät. Und die Scham gibt uns den Entwicklungsimpuls, aus dem die Kraft für Veränderung kommen kann.

\section{Würdevolle Pflege}

Würdevolle Pflege bedeutet, jemandem Demütigung zu ersparen. ${ }^{3}$ Wir sprechen manchmal davon, dass unwürdig ist, wie jemand leben muss oder unter welchen Bedingungen Pflege geleistet wird. Nach Stoecker fällt aber kein Mensch aus der Würde heraus, egal wie unselbstständig oder abhängig er oder sie Hilfe bedürfen zwar versorgt haben wollen, aber die Bedingungen dafür nicht bereitstellen? Dass wir wegschauen? Dass wir bei der Verteilung der Gelder zu wenig die Stimme erheben und es Leuten überlassen, die von der Pflege zu wenig Ahnung haben?

Wir tun gut daran, uns zu schämen, denn nur dann kann die Scham ihre schützende Funktion entfalten. Dann fühlen wir, dass die Grenze erreicht oder überschritten ist, in diesem Fall die Grenze des Zumutbaren und die Grenze des Aushaltbaren. Die Scham ist das Warnsignal, das anzeigt, wenn unsere fehlt. Die Würde wird verletzt, wenn etwa bei der Visite jemand entblößt wird, ohne auf den nötigen Sichtschutz zu achten. Oder wenn das Bedürfnis, von gleichgeschlechtlichen Pflegekräften versorgt zu werden, missachtet wird, obwohl dies möglich wäre. Strukturelle Bedingungen sind genauso wichtig für die Vermeidung überflüssiger Scham, zum Beispiel dass es Räume gibt, in denen Intimität geschützt ist. Es gibt unzählige Beispiele in der Pflege, bei denen deutlich wird, dass Fingerspitzengefühl, Wissen und Können gebraucht wird, um die Würde zu wahren die Würde ist eben antastbar. Und die Scham hütet diese Würde.

\section{Was Scham ist}

Scham betrifft uns alle. Sie ist universell. Scham gehört zu den Gefühlen, die sich früh in unserer Kindheit entwickeln. ${ }^{4}$ Sobald ein Ich-Bewusstsein entsteht, können wir sagen: „Ich schäme mich.“ Daran sehen wir, dass die Scham immer den ganzen Menschen betrifft. Wir reagieren auch körperlich auf Scham: werden rot, schwitzen und ziehen uns ganz in uns selbst zurück. Wie der Igel, der seine verletzliche Seite schonen muss, igeln wir uns ein und gehen aus dem Kontakt.

Im Zustand akuter Scham werden unsere Überlebensimpulse aktiv: fliehen oder verstecken, erstarren oder kämpfen. Aus diesen Überlebensimpulsen ist beispielsweise das Schlagen, Spucken und Beschimpfen einer Bewohnerin zu erklären, wenn sie sich gegen die Intimpflege wehrt, aber auch das starre Über-sich-ergehenLassen eines Bewohners, der an die Decke starrt und erst wieder „auftaut“, wenn er von den Ausscheidungen gesäubert ist, und das Weglaufen aus einer Situation, die peinlich ist, und Bewohner sich verstecken und erst wiederkommen, wenn die Scham nicht mehr so akut ist.

Warum wir Scham oft nicht wahrnehmen, sondern durch eine andere Reaktion ersetzen, hat einen einfachen Grund: die Scham ist eine der schmerzhaftesten Emotionen, die wir kennen. Darum wird sie mitunter verleugnet: „Nein, ich schäme mich überhaupt nicht!", oder verdrängt. Wenn sie verdrängt wird, wissen wir nichts mehr von ihrer Ursache, so zum Beispiel bei Opfern von Missbrauch, die 
Fallbeispiel: Frau Weiß - Herr Kaiser

In der Pflege müssen täglich Grenzen überschritten werden. Würdevoll Grenzen zu überschreiten, bedeutet, nicht zu beschämen oder, in anderen Worten, zusätzliche Demütigung zu ersparen. Wie schwierig das sein kann, mag ein Beispiel verdeutlichen. Frau Weiß ist Altenpflegerin und sieht, als sie nach Herrn Kaiser schaut, dass er in seinen Exkrementen liegt. Er hat sich nicht gemeldet, mal wieder aus Scham. Sie hat ihm schon ganz oft gesagt, dass er sich melden soll und dass er sich nicht zu schämen braucht. Jetzt passt es ihr gerade gar nicht, weil es bei ihm auch immer sehr lange dauert, ihn zu säubern. Er ist sehr unbeweglich und schmerzempfindlich. Sie ärgert sich, sagt ihm das und dass er sich nicht immer zu schämen brauche und sich einfach melden solle. Er blickt an die Decke und sagt gar nichts. Während sie ihn säubert, beeilt sie sich. Beide reden nicht und schämen sich. Sie sich für ihre Aggression und Ungeduld, er sich dafür, dass er ihr Arbeit macht. Sie hat ihn zusätzlich beschämt. Der Unterschied von Scham und Beschämung ist: die Scham ist die eigene Leistung, Beschämung wird uns von anderen zugefügt.

Dieses Beispiel soll verdeutlichen, dass wir Beschämung nicht immer ersparen können. Herr Kaiser findet es beschämend, so ausgeliefert und auf fremde Hilfe angewiesen zu sein. Wir können höchstens zusätzliche Scham ersparen, indem wir nicht noch zusätzlich beschämen. Aber auch das können wir nicht immer vermeiden, denn wir haben selbst auch unsere Grenzen. Wenn wir unsere Grenzen überschreiten müssen, verlieren wir unseren Schutz und die Würde kommt in Gefahr. Marks nennt vier Grundbedürfnisse, die unsere Würde schützen ${ }^{5,6}$ :

- Anerkennung

- Schutz

- Zugehörigkeit

- Integrität

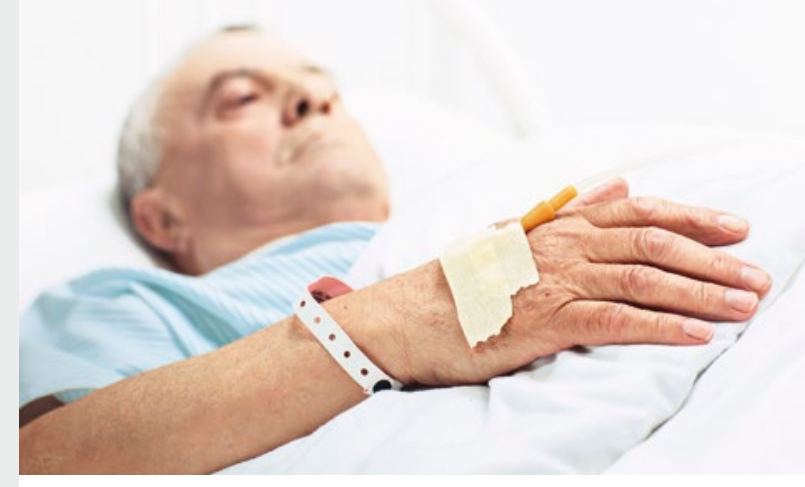

manchmal kein Bewusstsein dafür haben, was ihnen passiert ist.

Wir können aber auch so tun, als stünden wir über allem, und uns stolz und unnahbar zeigen, um uns vor zu viel Scham zu schützen. Oder wir stellen Schamlosigkeit zur Schau und suggerieren damit, dass uns alles gar nichts ausmachen würde. Es ist in der Pflege sehr wichtig, zu wissen, dass hinter der zur Schau gestellten Schamlosigkeit auch abgrundtiefe Scham stecken kann, die nur über die Flucht nach vorn ausgehalten werden kann. „Masken der Scham“ nennt Wurmser die Abwehrformen und er beschreibt noch viele weitere. ${ }^{4}$

Scham ist universell, aber auch individuell ausgeprägt, abhängig von unserer persönlichen, biografischen oder religiösen Prägung, unserem Milieu, unserem Alter und vielem mehr. Wofür die eine Person sich zutiefst schämt, das ist für andere eine kleine Peinlichkeit. Wir können niemals unser eigenes Schamempfinden auf andere übertragen. Die Scham kommt einfach und ist nicht wegzudiskutieren. Besser ist es, die Scham wahrzunehmen, die eigene und die von anderen, und damit zu sehen: Hier ist eine Grenze, die darf ich nur mit guten Gründen überschreiten, und wenn ich sie überschreite, muss ich das mit höchster Sorgfalt tun.

\section{Das Bedürfnis nach Anerkennung}

Unser Bedürfnis nach Anerkennung sagt: „Sieh mich!“ Wir brauchen es, als Personen wahrgenommen und geachtet zu werden. Das Gegenteil ist die Missachtung. Diese kann individuell geschehen, indem jemand übergangen, wie Luft behandelt oder bloßgestellt wird. Sie kann auch strukturell geschehen, wenn zum Beispiel in einer Gesellschaft Menschen, die nicht (mehr) produktiv sein können, nur noch als Kostenfaktor gesehen werden.

In der Pflege kann Anerkennung darum auf der individuellen Ebene gewährt werden, indem die Person, so, wie sie geworden ist, geachtet wird, und ihr zum Beispiel das Essen angeboten wird, das sie mag, ihre religiösen Bedürfnisse geachtet werden oder das „Du“ nur in therapeutisch sinnvollen Situationen in der Pflege Anwendung findet, etwa bei demenziell erkrankten Menschen, wenn damit der $\mathrm{Zu}$ gang ermöglicht wird.

Pflegekräfte können sich selbst und gegenseitig dafür Anerkennung geben, dass sie diese wichtige und großartige Arbeit machen, auch unter den gegebenen Bedingungen. Sie können ihre eigene Scham anerkennen und sie damit erkennen, sie achten und als Hüterin der Würde begreifen, nicht als Schwäche. Auch wenn sie dabei immer auch an ihre Grenzen kommen.

\section{Das Bedürfnis nach Schutz}

Während die Anerkennung sagt: „Sieh mich!“, sagt der Schutz: „Sieh nicht immer alles von mir, wahre meine Grenzen!“ Es geht hier um den Schutz vor Blicken anderer, zum Beispiel, indem Zimmertüren geschlossen werden, ein Sichtschutz angebracht wird, nur die Informationen weitergegeben werden, die nötig sind. Aber auch, indem die Grenzen erfühlt, erfragt oder gesehen werden, die eigenen und die der anderen Menschen und, soweit wie möglich, geachtet werden.

Das Bedürfnis nach Schutz ist auch das Bedürfnis der richtigen Nähe und Distanz. Wir Menschen sind in diesen Bedürfnissen unterschiedlich. Das eigene Empfinden auf andere zu übertragen, kann eine Grenzverletzung sein. Sensibel mit den Grenzen anderer umzugehen, bedeutet zuerst einmal, die Grenzen wahrzunehmen, sie zu reflektieren und dann so weit möglich, zu 
Integrität zu wahren, bedeutet, sich selbst in die Augen schauen zu können. Oder „nicht das Gesicht zu verlieren“, wie der Volksmund treffend ausdrückt (Symbolbild).

schützen. Jemandem die erwünschte Nähe zu gewähren, kann das eigene Bedürfnis nach Distanz verletzen. Nur wenn die Grenzen aller gewahrt sind, ist dem Bedürfnis nach Schutz Rechnung getragen. Es kann auch darum gehen, jemandem freundlich und unter Anerkennung der Person die eigenen Grenzen aufzuzeigen.

\section{Das Bedürfnis nach Zugehörigkeit}

Wie schmerzhaft es sein kann, nicht dazuzugehören, ausgegrenzt zu werden, wissen alle, die das schon erfahren haben. Das Bedürfnis nach Zugehörigkeit wah- den Rand gedrängt zu sein, nicht mithalten zu können. Alten Menschen, auch demenziell erkrankten Menschen zu vermitteln, dass sie dazugehören, dass sie ein ebenso geachtetes Mitglied der Gesellschaft sind wie andere, heißt, Zugehörigkeit zu vermitteln. Auch wenn Menschen sich gegenseitig ausschließen, können wir integrativ wirken, indem wir deutlich machen, dass alle dazugehören, auch die Kollegin, die einen Fehler gemacht hat, und auch der alte Mann, der in seiner unbändigen Wut die Teller durch den Saal geworfen hat.

durch Handlungen oder durch Unterlassungen, wenn wir Zeugen von Unrecht werden und nicht eingreifen oder wenn wir gegen unsere inneren Überzeugungen handeln.

Es ist die Integrität, die in Gefahr kommt, wenn Pflegekräfte, wie eingangs beschrieben, nicht entsprechend ihren Werten pflegen können. Eine Studie der FH Münster beschreibt das erschreckende Ergebnis, dass „über zwei Drittel der Befragten (Pflegekräfte) bemängeln, dass sie zur Erledigung der Aufgaben und für die Zuwendung zum Patienten nicht ausreichend Zeit haben und die anfallende Arbeit in der dafür vorgesehenen Zeit kaum zu schaffen ist."7 Diese hohe Zahl an Pflegekräften kann mit Schamgefühlen zurückbleiben, weil sie „etwas schuldig geblieben sind“, denen, für die sie zuständig sind, aber auch sich selbst. Hier kann es wichtig sein, für die eigene Integrität zu sorgen, indem Missstände an entsprechender Stelle angezeigt werden, sich für die Veränderung einzusetzen und sich zusammenzuschließen.

Diese vier Grundbedürfnisse zu wahren, ermöglicht würdevolles Miteinander. Sie müssen zuerst bei uns selbst und in unseren Teams gewahrt werden, damit sie auch im pflegetherapeutischen Handeln sichtbar werden können.

Ein wichtiger Bereich der Grenzverletzungen ist der Umgang mit Sexualität. Im folgenden Abschnitt wird kurz darauf eingegangen. ${ }^{8}$

\section{Sexualität, Erotik und Scham}

Sexualität und Erotik sind für uns Menschen elementar und wichtiger Teil unserer Lebenskraft. Sie sind darum auch aus Pflegesituationen nicht wegzudenken. Ich unterscheide drei Bereiche, die Pflegende

ren wir, wenn wir Dinge von uns verbergen. „Das sollst du von mir nicht sehen!“, zeigt manchmal an, dass wir Angst haben, dass uns andere ausschließen. In unserer Gesellschaft gehört man dazu, wenn man leistungsfähig, jung, gut aussehend ist.

Häufig haben diejenigen, die aus diesen Normen herausfallen, das Gefühl, an

\section{Das Bedürfnis nach Integrität}

Das Bedürfnis nach Integrität hilft uns, sensibel zu sein für die Übereinstimmung des Seins mit den eigenen Werten. Die aus der Verletzung resultierende Scham wird auch die „Gewissensscham“ genannt. Wir können dieses Grundbedürfnis verletzen, wenn wir unsere Werte verletzen, sei es professionell bewältigen müssen: ${ }^{9}$

- sexuelle Bedürfnisse als existenzieller Teil aller Menschen

- aufkommende Erotik und sexuelle Bedürfnisse, ausgelöst durch Pflegesituationen

- Umgang mit sexistischem Verhalten in der Pflegesituation 
Trunkenpolz et al. haben mittels Beobachtungen herausgefunden, dass vor allem Männer in Altenhilfeeinrichtungen in ihren sexuellen Bedürfnissen nicht nur nicht wahrgenommen, sondern noch beschämt werden. Ihre Bedürfnisse wahr- und ernstzunehmen und darauf professionell zu reagieren, wahrt die Würde der Bewohner und Bewohnerinnen und der Mitarbeiter. ${ }^{10}$

Während das Anklopfen, bevor ein Zimmer betreten wird, inzwischen zum Standard gehört, gibt es doch selten Räume, in welchen Intimität ungestört möglich ist. Ein Schild an der Zimmertür oder ein schöner Raum dafür und das Anbieten von ausgebildeten Sexualassistentinnen und -assistenten wären Möglichkeiten, sexuelle und erotische Gefühle in die Normalität zu holen und damit aus der Tabuzone herauszuführen.

Wenn vor allem weibliche Pflegekräfte mit sexistischen Äußerungen umgehen müssen, werden sie dafür nicht selten auch noch verantwortlich gemacht, nach dem Motto „So, wie du auftrittst!“. Es braucht ein kollegiales Klima, Möglichkeiten, diese Situationen im professionellen Rahmen zu besprechen, und klare Vorgehensweisen der Wertschätzung allen Beteiligten gegenüber. Diese Gefühlsarbeit zu beherrschen, ist Teil des professionellen Anspruchs an Pflege und darf nicht im Tabubereich der Negierung von Gefühlen verborgen bleiben.

\section{Zusammenfassung}

Eine würdevolle Pflege gelingt nur, wenn für die Pflege die Rahmenbedingungen stimmen. Anerkennung, Schutz, Vermitteln von Zugehörigkeit und Integrität sind die Merkmale würdevoller Pflege. Scham muss wahrgenommen und gelten gelassen werden, nur dann kann sie sich als Hüterin der Würde entfalten. Das geht aber nur, wenn mit der eigenen Scham, der Schambiografie, schamauslösenden Situationen und den Abwehrmechanismen, welche die Scham auslöst, bewusst und reflektiert umgegangen wird, wenn hinter der Aggression, der Erstarrung oder auch der Schamlosigkeit die Scham erfühlt und geachtet wird - bei sich selbst und bei anderen. Dafür müssen Räume geschaffen werden, in denen die Würde gewahrt werden

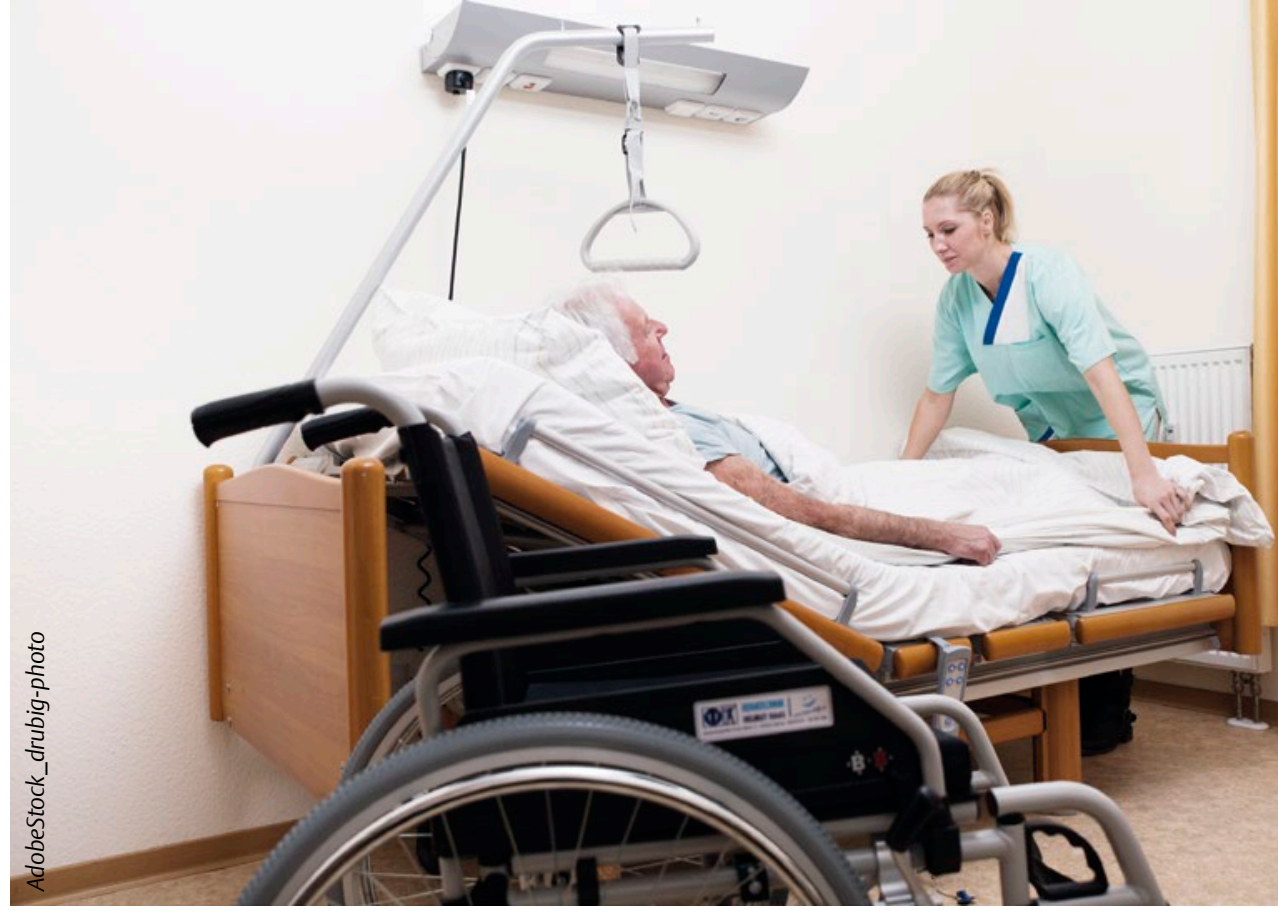

Der Umgang mit erotischen und sexuellen Gefühlen, die natürlich sind, auch bei Krankheit und im Alter, erfordert von Pflegekräften hohe Professionalität und Reflexionsfähigkeit (Symbolbild).

kann, seien es Räume für professionelle Reflexion und Supervision für Pflegekräfte, für ungestörte Privatsphäre bei Bewohnerinnen und Bewohnern oder Räume für würdevolle Pflege mit ausreichend und gut qualifiziertem Personal. Die Wahrung der Würde in Pflege und Gesellschaft geht uns alle an. Die Scham kann uns dabei beste Dienste leisten, die Bedingungen zu schaffen, damit sich bei denen, welche pflegen und welche Pflege in Anspruch nehmen (müssen), Räume der Würde entfalten können. Dann ist die Pflege das, was Florence Nightingale schon sagte: Kunst und Wissenschaft in der Arbeit mit dem Menschen als dem Tempel Gottes.

\section{Literatur}

1 Isfort M et al. Pflege-Thermometer 2016. Eine bundesweite Befragung von Leitungskräften zur Situation der Pflege und Patientenversorgung in der ambulanten Pflege. Herausgegeben von: Deutsches Institut für angewandte Pflegeforschung e. V. (dip), Köln, 2016. Online abrufbar unter www.dip.de

2 Aiken LH. Superior outcomes for Magnet Hospitals: The evidence base; Center for Health Outcomes and Policy Research. University of Pennsylvania School of Nursing, Washington DC; 2002

3 Stoecker R. Das höchste Gut? Menschenwürde aus moralphilosophischer Sicht. In: Dr. med. Mabuse 2017; 227 (Mai/Juni): 22-25

4 Wurmser L. Die Maske der Scham. Zur Psychoanalyse von Schamaffekten und Schamkonflikten. 3. Aufl. Berlin: Springer; 1997

5 Marks S. Die Würde des Menschen oder Der blinde Fleck in unserer Gesellschaft. Gütersloh: Gütersloher Verlagshaus; 2010
6 Marks S. Scham - die tabuisierte Emotion. 4. Aufl. Ostfildern: Patmos; 2013

7 Buxel H. Jobwahlverhalten, Motivation und Arbeitsplatzzufriedenheit von Pflegepersonal und Auszubildenden in Pflegeberufen. Ergebnisse dreier empirischer Untersuchungen und Implikationen für das Personalmanagement und -marketing von Krankenhäusern und Altenpflegeeinrichtungen. Studienbericht, FH Münster, 2011, S. 6; https://www.fh-muenster.de/oecotrophologie-facility-management/downloads/holger-buxel/2011_Studie_Zufriedenheit_Pflegepersonal. pdf; Zugriff: 29.01.2016

8 Zur vertiefenden Auseinandersetzung sei empfohlen: Immenschuh U, Marks S. Scham und Würde in der Pflege. Ein Ratgeber. Frankfurt: Mabuse-Verlag; 2014. Und: Immenschuh U. Würdevoll mit Schamgefühlen umgehen. In: Die Schwester, Der Pfleger 2016; 5: 38-41

9 Immenschuh (2016), s. Fußnote 8

10 Trunkenpolz K. et al. Von der Infant Observation zur Altersforschung: Die psychoanalytische Methode des Beobachtens nach dem Tavistock-Konzept im Kontext von Forschung. In: Zeitschrift für Individualpsychologie 2009; 34: 330-351

\section{Autorin}

Prof. Dr. Ursula Immenschuh

Gesundheits- und Krankenpflegerin, Dozentin in Aus-, Fort- und Weiterbildung, seit 2004 Professorin für Pflegepädagogik und Pflegewissenschaft an der Katholischen Hochschule Freiburg, Referentin zum Thema Würde und Scham in der Pflege

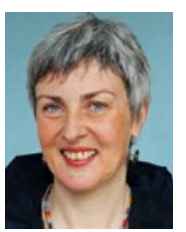

\section{Bibliografie}

DOI 10.1055/a-0598-9813

GGP 2018; 2: 115-119

(c) Georg Thieme Verlag KG

Stuttgart · New York · ISSN 2511-7548 